\title{
Antigen recognition in the pathogenesis of immunoglobulin A-related autoimmune bullous diseases
}

\author{
Zhiliang Li, Ke Jing, Suo Li, Suying Feng
}

Jiangsu Key Laboratory of Molecular Biology for Skin Diseases and STIs, Institute of Dermatology, Chinese Academy of Medical Sciences and Peking Union Medical College, Jiangsu, China

Adv Dermatol Allergol 2018; XXXV (4): 338-343

DOI: https://doi.org/10.5114/ada.2018.77663

\begin{abstract}
Immunoglobulin A (IgA) is the most common subtype of antibodies in mucosal surfaces. In most of autoimmune bullous diseases, however, immunoglobulin $G(\lg G)$ is the main pathogenic antibody that plays a role through complementation. The IgA antibody for epidermal connection protein can be found in the sera of some patients with blistering skin disease. Of these patients, some have the IgA antibody in their sera, while others have IgG and IgA antibodies. IgA-related autoimmune bullous diseases are less common in clinical practice. In the past, these diseases were not fully understood and their classifications were confusing. Recently, some progress has been made in the study of these diseases.
\end{abstract}

Key words: immunoglobulin A, autoimmune bullous disease.

\section{Introduction}

Immunoglobulin A ( $\lg \mathrm{A})$ is the most common subtype of antibodies in mucosal surfaces. In most of autoimmune bullous diseases, however, immunoglobulin G (IgG) is the main pathogenic antibody that plays a role through complementation. The IgA antibody targeting epidermal connection protein can be found in the sera of some patients with blistering skin disease. Of these patients, some have the IgA antibody in their sera, while others have IgG and IgA antibodies. IgA-related autoimmune bullous diseases are less common in clinical practice. In the past, these diseases were not fully understood and their classifications were confusing. Recently, some progress has been made in the study of these diseases.

\section{IgA pemphigus}

Immunoglobulin A (IgA) pemphigus is a subtype of pemphigus that exhibits reticular deposition of the IgA antibody in the intercellular space when observed through indirect immunofluorescence (DIF). Its clinical manifestations consist of blisters or pustules in the fold area. IgA pemphigus was previously divided into the following main types: intraepidermal neutrophilic IgA dermatosis (IEN) and subcorneal pustular dermatosis (SPD). By studying 49 IgA pemphigus patients, Hashimoto et al. added another three subtypes of IgA pemphigus [1, 2].
These subtypes, namely, pemphigus vulgaris, pemphigus foliaceus, and pustules subtypes, were categorized according to the clinical manifestations and different target antigens of the IgA antibodies. The antigen of IgA pemphigus is heterogeneous, and the target antigen of SPD subtype is desmocollin 1 [3]. Teye et al. [4] detected the titer of the anti-desmocollin 1 antibody in sera of patients through ELISA and by using mammalian-derived desmocollin 1 . They were able to achieve a positive rate of $85.7 \%$. The target antigens of the pemphigus vulgaris and pemphigus foliaceus subtypes are desmoglein 3 and desmoglein 1, respectively. Meanwhile, the target antigens of the IEN type and pustules subtype remain unknown. Moreover, in the sera of some IgA pemphigus patients, no anti-epidermal protein antibodies can be detected. The mechanism of the IgA body in the pathogenesis of IgA pemphigus remains unidentified.

\section{IgG/lgA pemphigus}

IgG/lgA pemphigus may be a result of the simultaneous occurrence of IgA pemphigus and pemphigus vulgaris (PV) or pemphigus foliaceus. Spaeth et al. [5] found that the anti-DSG3 IgA antibody can be detected in the sera of the $60 \%(9 / 15)$ of the acute-onset PV patients through the Western blot method. Furthermore, the anti-DSG3 IgA antibody can be detected in $72 \%(13 / 18)$

Address for correspondence: Suying Feng, Jiangsu Key Laboratory of Molecular Biology for Skin Diseases and STIs, Institute of Dermatology, Chinese Academy of Medical Sciences and Peking Union Medical College, 12 Jiangwangmiao St, Nanjing, Jiangsu 210042, China, phone: +86 15298384673, e-mail: fengsuying2010@163.com Received: 14.03.2017, accepted: 27.06.2017. 
of the chronic active PV. Notably, IgA antibodies cannot be detected in skin lesions through direct immunofluorescence. Mentink et al. [6] found that anti-DSG1 and anti-DSG3 antibodies can be detected in 22\% (22/100) and $41 \%(41 / 100)$ of the sera of the pemphigus patients, while the percentage of IgA antibodies detected in the skin lesions of these patients through direct immunofluorescence was $17.9 \%$ (7/39). In these two studies, the patients had typical clinical manifestations of pemphigus. Thus, the IgA antibodies detected in these studies may be a concomitant phenomenon. Typical IgG/lgA pemphigus is relatively rare, and only 20 cases have been reported so far. The clinical manifestations in these cases are diverse and characterized by pustules or annular blisters [7]. In our own clinic, we encountered a case of pustular pemphigus with IgG and IgA antibodies detected by DIF and ELISA, and the titer of Dsg1 IgA was related to the disease activity (Figure 1) [8]. Some studies suggested that IgG/lgA pemphigus may be associated with internal tumors, such as adenocarcinoma of the endometrium and lung, ovarian, and gallbladder cancers [9]. In our own study, of the six cases of drug-induced pemphigus investigated, four had the anti-DSG1 IgA antibody. The titers of the anti-DSG1 antibodies obtained from these four patients decreased when the conditions of these patients improved. Thus, the drug may also be a predispos-
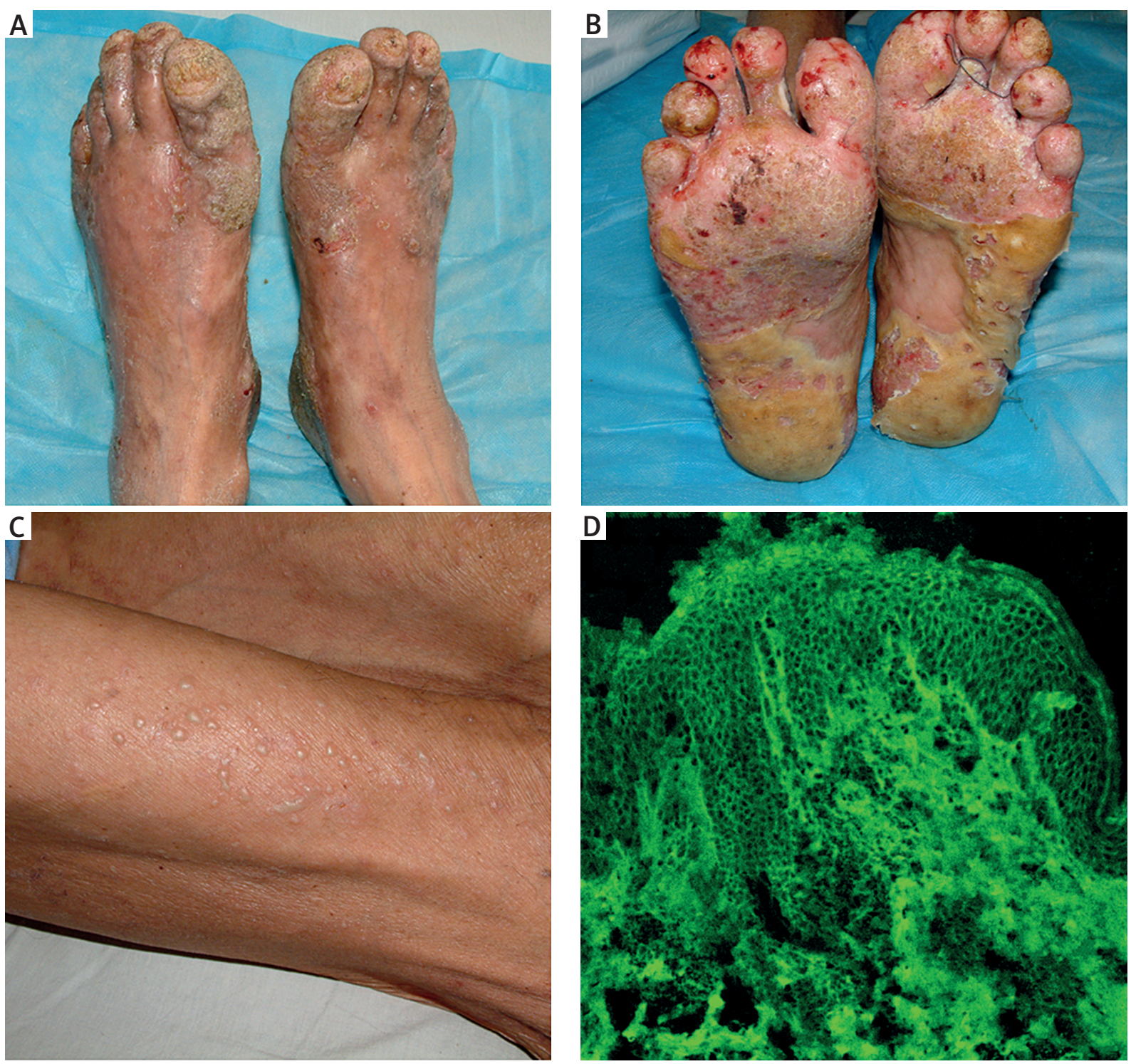

Figure 1. A - Pustules on the dorsum of the feet and toes, B - papulovesicles and pustules on the plantar surface of feet and between the toes, $\mathbf{C}$ - vesicles and pustules on the lower limb, D - direct immunofluorescence showing IgA fish-net like deposits in the epidermis (original magnification 20x) 
ing factor for lgG/lgA pemphigus, except in cases associated with tumor [10]. The most common target antigen detected by ELISA in the sera of the IgG/IgA pemphigus patients is DSG1. Anti-DSG3, anti-desmocollin, and antidesmoplakin antibodies were also detected in the sera of some patients [11]. Despite these findings, the pathogenesis of IgG/lgA pemphigus and whether IgA or IgG antibody causes blisters in this disease remain unknown.

\section{Dermatitis herpetiformis}

Dermatitis herpetiformis $(\mathrm{DH})$ is the skin manifestation of gluten sensitivity, and in most patients it is associated with gluten-sensitive enteropathy. The IgA type of anti-endomysial antibody (EMA) and anti-tissue transglutaminase (tTG) antibody can be detected in the sera of the DH patients. In addition, IgA antibodies deposited as granules in the dermal papilla layer of their skin lesions. These granules were detected by direct immunofluorescence. The disease is a genetic susceptibility and has more occurrences in the Caucasian population than in the Eastern population. However, Zhang et al. [12] reported 22 cases of DH in the Chinese population. In the lesions of most of these patients, granular IgA antibodies were detected in the papillary layer through direct immunofluorescence. Meanwhile, the positive rates of anti-EMA and anti-tTG antibodies in the Chinese population are lower than those in the Caucasian population, of which nearly all patients had HLA DQ2 or HLA-DQ8 alleles. The occurrence of DH in the Chinese population was found to be associated with HLA B*0801 and DRB1 ${ }^{\star} 0301$ [13]. The target antigen of $\mathrm{DH}$ is epidermal transglutaminase (eTG) [14]. In the sera of the DH patients, immune complexes formed by the IgA antibodies and eTGs were detected and IgA anti-eTG antibodies were present in DH sera either unbound or in immune complexes [15]. This immune complex can be co-deposited with the fibrinogen at the dermal-epidermal junction [16]. This complex then recruits macrophages and neutrophils to initiate the fibrinolytic process, which results in blisters.

\section{Lamina lucida-type linear IgA bullous dermatosis}

Linear IgA bullous dermatosis (LABD) is categorized into lamina lucida and sublamina densa types according to the deposition position of the IgA antibody in the saltsplit skin indirect immunofluorescence (SS-IIF) [17]. The classic LABD often refers to lamina lucida, which is the most common type. The sublamina densa-type is a rare disease, and most cases of this type are IgA-EBA (see the sixth section "Sublamina densa-type IgA bullous dermatosis"). Lamina lucida-type LABD mostly occurs in children and can be induced by drugs. Its typical manifestation is characterized by scattered or generalized circular blisters.
In addition, as a characteristic manifestation of the disease, IgA antibody deposition is observed in the basement membrane in a linear manner, as indicated by DIF. In the sera of some patients, the circulating IgA antibody deposits on the epidermal side and can be detected through SS-IIF. The positive rates of the serological tests varied widely in different studies. The target antigen of lamina lucida-type LABD was LAD97 and LAD120 in previous research, and both antigens are pyrolysis products of BP180. The antibody of LABD binds with the NC16A domain of BP180. In contrast to bullous pemphigoid (BP), the antigen epitope is not in the MCW-1 of NC16A, but in the location where NC16A is attached to collagen 15. This antigen epitope is not present in an intact BP180. At instances when the extracellular segment of BP180 ruptures, the folding site of NC16A and collagen 15 forms new epitopes, which may serve as binding sites for IgA antibodies in lamina lucida-type LABD [18]. The pathogenesis of LABD is different from BP because the ability of IgA antibodies to activate complements is extremely weak. Thus, IgA antibodies cannot play a pathogenesis role by activating complements. Fc receptors (FcoRI) can connect to IgA antibodies and can be detected on the surface of granulocytes. The pathogenic IgA antibodies in the sera of LABD patients bind with the corresponding antigens and form immune complexes, which can recruit granulocytes and bind with the FcaRIs on surfaces of the granulocytes. The granulocytes are then activated, thereby releasing chemokines. The process continues through a positive feedback mechanism and thus granulocytes are continuously recruited to the epidermaldermal junction [19]. The recruited granulocytes then release proteases, which cause the subepidermal blisters. In in vitro experiments, the specific inhibition of FcaRIs can prevent pathogenic IgA antibody-induced tissue damage, and this finding have implications for the treatment of LABD [20].

\section{Linear IgA/lgG bullous dermatosis}

Patients with both IgA and IgG-type anti-basement membrane zone (BMZ) antibodies detected by DIF or serological assays are often diagnosed to have linear IgA/ IgG bullous dermatosis (LAGBD). In previous studies, because of different diagnostic criteria for the disease, some LAGBD cases were classified into BP categories, while some were classified into LABD categories. Through different detection methods, the percentages of IgA anti$\mathrm{BMZ}$ antibodies detected in BP patients reported in the past were $25.8 \%$ [21], 63.3\% [22], 8.2\% [23], and 87.5\% [24]. The percentages of IgG antibodies detected in LABD patients were 53.5\% [25], 13.0\% [26], 16.1\% [27], 21.1\% [28], 69.1\% [29], and 77.3\% [24]. Children with LABD are more likely to develop coexisting IgG antibodies [28]. These data show that LAGBD is not rare, and increasing the accuracy of the detection method can increase the number of cases detected. Clinical manifestations 
of LAGBD may be related to the type of the antibody, that is, when patients mostly have pathogenic IgG antibodies, they show BP symptoms. Conversely, when they mostly have IgA, they exhibit LABD symptoms. However, many patients exhibit atypical clinical manifestations and thus their conditions are difficult to categorize into $B P$ or $L A B D$. Therefore, BP, LAGBD, and LABD are likely spectrum diseases. $B P$ and $\angle A B D$ are located at the extremes of the spectrum, while LAGBD is in the intermediate state. Allen and Wojnarowska [30] revealed that the target antigen of the IgA antibody tends to be diversified and more likely to exhibit epitope spreading. In recent years, an increasing number of cases have supported this conclusion. The clinical manifestations in these cases are atypical, and thus these cases are difficult to classify into typical BP, LABD, EBA, or MMP. Furthermore, the anti$B M Z$ antibodies detected in the sera of these patients are against a variety of different epitopes, such as LAD120 [31], N-terminal, or C-terminal of BP180 [32], BP230 [32], laminin $\alpha 3$, laminin $\beta 3$, laminin $\gamma 2$ [33], type VII collagen [32], laminin $\gamma 1$ [32], integrin $\alpha 6$ [33], integrin $\beta 4$ [33], and NC16A [34]. The diversity of the target antigen may be related to epitope spreading. As significant differences are present among LAGBD patients, large-sample studies are necessary to determine whether or not IgA or IgG antibodies induce blisters.

\section{Sublamina densa-type IgA bullous dermatosis}

Dermal side IgA deposition can be detected using SS-IIF in sublamina densa-type IgA bullous dermatosis. Some of the target antigens through this method are type VII collagen, laminin $\gamma 1$, and laminin 332. Tsuchisaka et al. [35] reported 12 cases of sublamina densa-type IgA bullous dermatosis, and most of the sera of the patients have a positive result in IIF when frozen sections of the normal skin were used as substrates. Negative results in IIF were obtained when frozen sections of the skin of recessive dystrophic epidermolysis bullosa patients were used. The target antigen of most sublamina densa-type IgA bullous dermatosis is type VII collagen and this entity can be renamed as IgA-EBA. Given the low sensitivity of serological tests, a simple and cheap differentiation method based on Vodegel et al. $[36,37]$ can be used to differentiate IgA-EBA from lamina lucida-type LABD. In lucida-type $L A B D$, the pattern of IgA deposition along DEJ should be "N-serrated" contrary to IgA-EBA in which this pattern should be "U-serrated". Besides, fluorescence overlay antigen mapping using laser scanning confocal microscopy (FOAM-LSCM) might be also a useful tool to differentiate between these two entities [37]. Further research revealed that the antigen epitopes of these patients are located at the conformational epitopes in the collagenous domain of COL7 but not in the NC1 and NC2 domains which are the antigen epitopes of EBA [35]. Tsuchisaka et al. [35] also found a special case of sublam- ina densa-type IgA bullous dermatosis, wherein the IgA antibodies in serum can bind to 105KD-laminin $\gamma 2$ and type VII collagen. Anti-laminin $\gamma 1$ pemphigoid is a newly found subepidermal blister disease characterized by the presence of IgG antibodies against laminin $\gamma 1$. Most of the antigens are located at the c-terminus of laminin $\gamma 1$. Thus, only 1 case of sublamina densa-type IgA bullous dermatosis manifested by laminin $\gamma 1$ as target antigen was reported [38, 39]. However, the antigen epitope of IgA antibody is not located at the C-terminus of laminin $\gamma 1$, and the exact target spot and pathogenesis remains unclear. Zenke et al. [40] reported a case of sublamina densa-type IgA bullous dermatosis, the target antigen of which is laminin 332. The patient was induced intravenously by vancomycin, and the immunoblot showed that the IgA antibodies reacted with $145 \mathrm{kD}$ and $165 \mathrm{kD}$ laminin $\alpha 3$. The result of immunoblotting turned negative upon disease remission. The antilaminin $\alpha 3$ IgA antibody was pathogenic, but its exact target epitope and pathogenic mechanism still require further research.

\section{IgA-related mucous membrane pemphigoid}

In mucous membrane pemphigoid (MMP) patients, the presence of IgA antibodies alone is relatively rare in the absence of IgG antibodies [41]. In some patients with only mucosal involvement, only IgA antibody can be found in DIF; a diagnosis of LABD might have been previously established [42]. According to Chan et al. [43], these cases are more appropriate for the diagnosis of MMP. IgG antibodies are simultaneously present in the serum of most IgA-related MMP patients. By using different detection methods, the previously reported percentages of IgA antibodies detected in MMP patients were 63.2\% (DIF) [44], 53.7\% (IIF) [45], 77.8\% (immunoblotting using extracellular portion of BP180 as a substrate) [46], 62.9\% (DIF) [47], 50.8\% (immunoblotting using different cleavage fragments of BP180 as a substrate) [47], 36\% (ELISA using NC16a as substrate) [48], 44.0\% (SS-IIF) [49], $27.3 \%$ (DIF) [50]. In an ocular MMP study, the positive rate of IgA antibody detected by DIF was 23.5\% [51]. In another pure ocular MMP study, the positive rates of IgA antibody targeting integrin $\alpha 6$ and integrin $\beta$ detected by immunoblotting were $23.3 \%$ and $20.9 \%$, respectively [52]. The target antigens of IgA antibodies reported in MMPs include LAD-1 [44, 47], LABD97 [47], ectodomain of BP180 [46], intracellular domain of BP180 [44], NC16A [44], integrin $\alpha 6$ [53], integrin $\beta 4$ [53], laminin $\alpha 3$, laminin $\beta 3$, and laminin $\gamma 2[54,55]$. MMPs with IgA antibodies tend to be relatively severe $[45,47]$, and some cases involved laryngeal, pharyngeal [45, 47], and even severe esophageal stricture [52]. These MMP patients are often more resistant to treatment [56]. At present, the cause and pathogenesis of IgA antibody in MMP patients is not clear. The seriousness of the disease and the difficulty of treatment make it very urgent to study the mechanism of IgA production in MMP. 
Therefore, there are many kinds of IgA-related autoimmune bullous diseases, and some are rare. Research on these diseases is limited. Consequently, systematic classification is inadequate. These diseases are quite different from corresponding IgG-related bullous disease with regard to target antigen epitopes and pathogenic mechanisms. An in-depth study of these diseases can improve the understanding of the pathogenesis of bullous diseases and lead to the discovery of new effective treatments.

\section{Acknowledgments}

This work was supported by the grant from the Natural Science Foundation of Jiangsu (Grant No. BK20160153), Natural Science Foundation of China (Grant No. 81602781) and Basic Research Foundation of PUMC (Grant No. 2016ZX320014).

\section{Conflict of interest}

The authors declare no conflict of interest.

\section{References}

1. Hashimoto T, Teye K, Ishii N. Clinical and immunological studies of 49 cases of various types of intercellular IgA dermatosis and 13 cases of classical subcorneal pustular dermatosis examined at Kurume University. Br I Dermatol 2017; 176: 168-75.

2. Hashimoto T, Nishikawa T. Nomenclature for diseases with IgA antikeratinocyte cell surface autoantibodies. Br J Dermatol 2015; 173: 868-9.

3. Hashimoto T, Kiyokawa C, Mori O, et al. Human desmocollin 1 (Dsc1) is an autoantigen for the subcorneal pustular dermatosis type of IgA pemphigus. J Invest Dermatol 1997; 109: 127-31.

4. Teye K, Numata S, Ohzono A, et al. Establishment of IgA ELISAs of mammalian recombinant proteins of human desmocollins 1-3. J Dermatol Sci 2016; 83: 75-7.

5. Spaeth S, Riechers R, Borradori L, et al. IgG, IgA and IgE autoantibodies against the ectodomain of desmoglein 3 in active pemphigus vulgaris. Br J Dermatol 2001; 144: 1183-8.

6. Mentink LF, de Jong MC, Kloosterhuis GJ, et al. Coexistence of IgA antibodies to desmogleins 1 and 3 in pemphigus vulgaris, pemphigus foliaceus and paraneoplastic pemphigus. Br J Dermatol 2007; 156: 635-41.

7. Moriuchi R, Ito T, Kikuchi K, et al. Crusted impetigo-like lesion on the face: a case of IgG/lgA pemphigus. J Eur Acad Dermatol 2017; 31: e289-90.

8. Feng SY, Zhi L, Jin PY, et al. A case of IgA/lgG pustular pemphigus. Int J Dermatol 2012; 51: 321-4.

9. Lane N, Parekh P. IgG/IgA pemphigus. Am J Dermatopathol 2014; 36: 1002-4.

10. Feng S, Zhou W, Zhang J, et al. Analysis of 6 cases of druginduced pemphigus. Eur J Dermatol 2011; 21: 696-9.

11. Hosoda S, Adachi A, Suzuki M, et al. Case of pemphigus with immunoglobulin $\mathrm{G}$ and $\mathrm{A}$ antibodies, binding to both the intercellular spaces and basement membrane zone. I Dermatol 2016; 43: 194-6.

12. Zhang F, Yang B, Lin Y, et al. Dermatitis herpetiformis in China: a report of 22 cases. J Eur Acad Dermatol Venereol 2012; 26: 903-7.
13. Sun $Y$, Lin $Y$, Yang $B$, et al. The HLA alleles $B^{*} 0801$ and DRB1 ${ }^{\star} 0301$ are associated with dermatitis herpetiformis in a Chinese population. J Invest Dermatol 2016; 136: 530-2.

14. Sardy M, Karpati S, Merkl B, et al. Epidermal transglutaminase (TGase 3) is the autoantigen of dermatitis herpetiformis. J Exp Med 2002; 195: 747-57.

15. Gorog A, Nemeth K, Kolev K, et al. Circulating transglutaminase 3-immunoglobulin A immune complexes in dermatitis herpetiformis. J Invest Dermatol 2016; 136: 1729-31.

16. Gorog A, Nemeth K, Szabo L, et al. Decreased fibrinolytic potential and morphological changes of fibrin structure in dermatitis herpetiformis. J Dermatol Sci 2016; 84: 17-23.

17. Guide SV, Marinkovich MP. Linear IgA bullous dermatosis. Clin Dermatol 2001; 19: 719-27.

18. Yamauchi T, Matsushita S, Hashimoto T, et al. Major cleavage-dependent epitopes for linear IgA bullous dermatosis are formed at the boundary between the non-collagenous $16 \mathrm{~A}$ and collagenous 15 domains of BP180. J Dermatol Sci 2014; 76: 25-33.

19. van der Steen L, Tuk CW, Bakema JE, et al. Immunoglobulin A: Fc(alpha)RI interactions induce neutrophil migration through release of leukotriene B4. Gastroenterology 2009; 137: 2018-29.

20. van der Steen LP, Bakema JE, Sesarman A, et al. Blocking Fcalpha receptor I on granulocytes prevents tissue damage induced by IgA autoantibodies. J Immunol 2012; 189: 1594601.

21. Csorba K, Schmidt S, Florea F, et al. Development of an ELISA for sensitive and specific detection of IgA autoantibodies against BP180 in pemphigoid diseases. Orphanet J Rare Dis 2011; 6: 31 .

22. Horvath B, Niedermeier A, Podstawa E, et al. IgA autoantibodies in the pemphigoids and linear IgA bullous dermatosis. Exp Dermatol 2010; 19: 648-53.

23. Cozzani E, Drosera M, Parodi A, et al. Frequency of IgA antibodies in pemphigus, bullous pemphigoid and mucous membrane pemphigoid. Acta Derm Venereol 2004; 84: 381-4.

24. Kromminga A, Scheckenbach C, Georgi M, et al. Patients with bullous pemphigoid and linear IgA disease show a dual IgA and IgG autoimmune response to BP180. J Autoimmun 2000; 15: 293-300.

25. Ohata C, Ishii N, Koga H, et al. A clinical and serological study of linear IgA bullous dermatosis without linear immunoglobulin deposition other than IgA at the basement membrane zone in direct immunofluorescence. Br J Dermatol 2017; 177: 152-7.

26. Lings K, Bygum A. Linear IgA bullous dermatosis: a retrospective study of 23 patients in Denmark. Acta Derm Venereol 2015; 95: 466-71.

27. Monia K, Aida K, Amel K, et al. Linear IgA bullous dermatosis in Tunisian children: 31 cases. Indian J Dermatol 2011; 56: 153-9.

28. Horiguchi Y, Ikoma A, Sakai R, et al. Linear IgA dermatosis: report of an infantile case and analysis of 213 cases in Japan. J Dermatol 2008; 35: 737-43.

29. Allen J, Wojnarowska F. Linear IgA disease: the IgA and IgG response to dermal antigens demonstrates a chiefly IgA response to LAD285 and a dermal 180-kDa protein. Br J Dermatol 2003; 149: 1055-8.

30. Allen J, Wojnarowska F. Linear IgA disease: the IgA and IgG response to the epidermal antigens demonstrates that intermolecular epitope spreading is associated with IgA rather than IgG antibodies, and is more common in adults. Br J Dermatol 2003; 149: 977-85. 
31. Shimizu S, Natsuga K, Shinkuma S, et al. Localized linear IgA/lgG bullous dermatosis. Acta Derm Venereol 2010; 90: 621-4.

32. Sakaguchi M, Bito T, Oda Y, et al. Three cases of linear IgA/ IgG bullous dermatosis showing IgA and IgG reactivity with multiple antigens, particularly laminin-332. JAMA Dermatol 2013; 149: 1308-13.

33. Li X, Tsuchisaka A, Qian H, et al. Linear IgA/lgG bullous dermatosis reacts with multiple laminins and integrins. Eur J Dermatol 2015; 25: 418-23.

34. Kanayama Y, Tsuruta D, Tateishi C, et al. Two cases of infantile linear immunoglobulin A/immunoglobulin $\mathrm{G}$ bullous dermatosis. J Dermatol 2012; 39: 176-8.

35. Tsuchisaka A, Ohara K, Ishii N, et al. Type VII collagen is the major autoantigen for sublamina densa-type linear IgA bullous dermatosis. J Invest Dermatol 2015; 135: 626-9.

36. Vodegel RM, Jonkman MF, Pas HH, et al. U-serrated immunodeposition pattern differentiates type VII collagen targeting bullous diseases from other subepidermal bullous autoimmune diseases. Br J Dermatol 2004; 151: 112-8.

37. Zborowski T. Methods of diagnosis of autoimmune bullous dermatoses from the point of view of laboratory diagnostician. Dermatol Klin 2006; 3: 189-96.

38. Wozniak K, Hashimoto T, Ishii N, et al. Fluorescence overlay antigen mapping using laser scanning confocal microscopy differentiates linear IgA bullous dermatosis from epidermolysis bullosa acquisita mediated by IgA. Br J Dermatol 2013; 168: 634-8.

39. Wozniak K, Hashimoto T, Fukuda S, et al. IgA anti-p200 pemphigoid. Arch Dermatol 2011; 147: 1306-10.

40. Zenke Y, Nakano T, Eto H, et al. A case of vancomycin-associated linear IgA bullous dermatosis and IgA antibodies to the alpha3 subunit of laminin-332. Br J Dermatol 2014; 170: $965-9$.

41. Torchia D, Caproni M, Fabbri P. Linear IgA disease and desquamative gingivitis: time for inclusion in mucous membrane pemphigoid. Oral Dis 2008; 14: 768-9.

42. Di Zenzo G, Carrozzo M, Chan LS. Urban legend series: mucous membrane pemphigoid. Oral Dis 2014; 20: 35-54.

43. Chan LS, Ahmed AR, Anhalt GJ, et al. The first international consensus on mucous membrane pemphigoid: definition, diagnostic criteria, pathogenic factors, medical treatment, and prognostic indicators. Arch Dermatol 2002; 138: 370-9.

44. Schmidt E, Skrobek C, Kromminga A, et al. Cicatricial pemphigoid: IgA and IgG autoantibodies target epitopes on both intra- and extracellular domains of bullous pemphigoid antigen 180. Br J Dermatol 2001; 145: 778-83.

45. Setterfield J, Shirlaw PJ, Kerr-Muir M, et al. Mucous membrane pemphigoid: a dual circulating antibody response with IgG and IgA signifies a more severe and persistent disease. Br J Dermatol 1998; 138: 602-10.

46. Christophoridis S, Budinger L, Borradori L, et al. IgG, IgA and IgE autoantibodies against the ectodomain of BP180 in patients with bullous and cicatricial pemphigoid and linear IgA bullous dermatosis. Br J Dermatol 2000; 143: 349-55.

47. Oyama N, Setterfield JF, Powell AM, et al. Bullous pemphigoid antigen II (BP180) and its soluble extracellular domains are major autoantigens in mucous membrane pemphigoid: the pathogenic relevance to HLA class II alleles and disease severity. Br J Dermatol 2006; 154: 90-8.

48. Ali S, Kelly C, Challacombe SJ, et al. Salivary IgA and IgG antibodies to bullous pemphigoid 180 noncollagenous domain 16 a as diagnostic biomarkers in mucous membrane pemphigoid. Br J Dermatol 2016; 174: 1022-9.
49. Murakami H, Nishioka S, Setterfield J, et al. Analysis of antigens targeted by circulating IgG and IgA autoantibodies in 50 patients with cicatricial pemphigoid. J Dermatol Sci 1998; 17: 39-44.

50. Kanwar AJ, Vinay K, Sawatkar GU, et al. Clinical and immunological study of mucous membrane pemphigoid in a cohort of Indian patients. Int J Dermatol 2016; 55: e557-61.

51. Mehra T, Guenova E, Dechent F, et al. Diagnostic relevance of direct immunofluorescence in ocular mucous membrane pemphigoid. J Dtsch Dermatol Ges 2015; 13: 1268-74.

52. Jakubowska B, Kowalewski C, Ishii N, et al. Mucous membrane pemphigoid with severe stricture of the esophagus mediated by IgG and IgA autoantibodies to LAD-1. Eur I Dermatol 2015; 25: 510-2.

53. Li X, Qian H, Sogame R, et al. Integrin $\beta 4$ is a major target antigen in pure ocular mucous membrane pemphigoid. Eur J Dermatol 2016; 26: 247-53.

54. Hayashi I, Shinkuma S, Shimizu S, et al. Mucous membrane pemphigoid with generalized blisters: IgA and IgG autoantibodies target both laminin-332 and type XVII collagen. $\mathrm{Br}$ J Dermatol 2012; 166: 1116-20.

55. Hanawa F, Harada K, Andou N, et al. Case of mucous membrane pemphigoid characterized by circulating immunoglobulin $\mathrm{A}$ and immunoglobulin $\mathrm{G}$ autoantibodies to the gamma-2 subunit of laminin-332. J Dermatol 2012; 39: 962-3.

56. He Y, Shimoda M, Ono Y, et al. Persistence of autoreactive IgA-secreting B cells despite multiple immunosuppressive medications including rituximab. JAMA Dermatol 2015; 151: 646. 